Europhys. Lett., 26 (6), pp. 413-418 (1994)

\title{
Crossover from Self-Similar to Self-Affine Structures in Percolation.
}

\author{
E. Frey (*), U. C. TÄUBER (**) and F. SchWABL $\left(^{*}\right)$ \\ (*) Institut für Theoretische Physik, Physik-Department der Technischen Universität \\ München - James-Franck-Str., D-85747 Garching, Germany \\ (**) Lyman Laboratory of Physics, Harvard University - Cambridge, MA 02138, USA
}

(received 3 January 1994; accepted in final form 24 March 1994)

PACS. 05.20 - Statistical mechanies.

PACS. 05.40 - Fluctuation phenomena, random processes, and Brownian motion.

PACS. 64.60 - General studies of phase transitions.

\begin{abstract}
We study the crossover from self-similar scaling behavior to asymptotically self-affine (anisotropic) structures. As an example, we consider bond percolation with one preferred direction. Our theory is based on a field-theoretical representation, and takes advantage of a renormalization group approach designed for crossover phenomena. We calculate effective exponents for the connectivity describing the entire crossover region from isotropic to directed percolation, and predict at which scale of the anisotropy the crossover should occur. We emphasize the broad range of applicability of our method.
\end{abstract}

Scale-invariance appears in a great variety of physical problems and processes [1]. The simplest scaling laws are isotropic, and thus describe self-similar structures; on the other hand, in growth models frequently self-affine clusters emerge, which we consider as defined by anisotropic scaling. The important point now is that whether a structure appears self-similar or self-affine is always a matter of scale, i.e.: a structure, which on a large length scale is characterized by anisotropic-scaling laws, may be indistinguishable from a self-similar entity on sufficiently small scales.

Perhaps the most simple growth models incorporating these features are provided by percolation [2]. In ordinary, isotropic percolation sites or bonds are filled at random with equal probability $p$, and the emerging clusters are self-similar. However, in directed percolation [3], there is one distinct preferred direction (which we shall call the $t$-direction) with a bias along the positive $t$-direction, and anisotropic-scaling laws apply. If the effective anisotropy parameter $g$ is low, one expects almost isotropic-scaling behavior in a large region of parameter space, and only if the percolation threshold $p_{\mathrm{c}}$ is approached the asymptotic self-affine wil become apparent, if one views the system at very large length scales.

Our aim is to provide a quantitative description of the crossover from isotropic to directed percolation, and to calculate the characteristic anisotropy scale, at which the scaling behavior turns over from self-similarity to self-affinity. For these issues, the central quantity of interest is the pair correlation function $G\left(r_{2}, r_{1}\right)$, which measures the probability that sites $r_{2}=\left(x_{2}, t_{2}\right)$ and $r_{1}=\left(x_{1}, t_{1}\right)$ belong to the same cluster. Lines of constant $G$ describe the average shape of the percolating structure. Following the work of Cardy et al. [4,5], we apply their mapping of the statistical percolation problem onto a field-theoretical representation. The final result for $G^{0}$ (the superscript «0" denotes unrenormalized 
quantities $)$ is a sum $G^{0}\left(\boldsymbol{r}_{2} ; \boldsymbol{r}_{1}\right)=\sum_{m, n=1}^{\infty}\left[(-i)^{m+n-2} / m ! n !\right] G_{m n}^{0}\left(\boldsymbol{r}_{2} ; \boldsymbol{r}_{1}\right)$, where

$$
G_{m n}^{0}\left(\boldsymbol{r}_{2} ; \boldsymbol{r}_{1}\right)=P \int \mathscr{D}\left[\phi_{0}, \tilde{\phi}_{0}\right] \phi_{0}\left(\boldsymbol{r}_{2}\right)^{m} \tilde{\phi}_{0}\left(\boldsymbol{r}_{1}\right)^{n} \exp \left[-\mathscr{T}\left[\phi_{0}, \tilde{\phi}_{0}\right]\right]
$$

$P$ is an operator projecting out those contributions which violate "causality", and the probability measure explicitly reads $[4,5]$

$$
\mathscr{T}=\int \mathrm{d}^{D} x \int \mathrm{d} t\left\{\tilde{\phi}_{0}\left[r_{0}-\nabla^{2}-\frac{1}{c_{0}^{2}} \partial_{t}^{2}+\frac{2 g_{0}}{c_{0}} \partial_{t}\right] \dot{\phi}_{0}+\frac{u_{0}}{2}\left[\tilde{\phi}_{0}^{2} \dot{\phi}_{0}-\tilde{\phi}_{0} \dot{\phi}_{0}^{2}\right]\right\},
$$

with $r_{0} \propto p-p_{\mathrm{c}}$. The anisotropy of the underlying percolation problem is reflected in the parameter $g_{0}$. For $g_{0}=0$, the problem is symmetric with respect to inversion of the lattice $\boldsymbol{r} \rightarrow-\boldsymbol{r}$, which corresponds to isotropic bond percolation [5]. For any non-zero $g_{0}$ the inversion symmetry is broken in the $t$-direction. A scaling analysis shows that $g_{0}$ grows under rescaling. For $g_{0} \rightarrow \infty$, with $c_{0} \rightarrow \infty$ such that $g_{0} / c_{0}$ remains finite, one arrives at reggeon field theory describing directed bond percolation [4]. On the basis of eqs. (1), (2) the perturbation expansion [6,7] with respect to the nonlinearity $u_{0}$ may be constructed.

In order to derive the anomalous dimensions leading to non-Gaussian behavior we proceed to study the ultraviolet divergences at the upper critical dimension $d_{c}$. In the dimensional regularization scheme [8] these UV singularities appear as poles $\propto 1 /\left(d-d_{c}\right)$. The technical difficulty here is now that these poles will be different in the two limiting cases $g_{0}=0$ and $g_{0} \rightarrow \infty$. In addition, via a simple rescaling of the model one finds that the upper critical dimensions for isotropic (I) and directed (D) percolation differ, namely $d_{\mathrm{c}}^{\mathrm{I}}=6$ and $d_{\mathrm{c}}^{\mathrm{D}}=5$, respectively. Thus, the «traditional» procedure of investigating the scaling behavior near one of the fixed points in the framework of a $\left(d_{c}-d\right)$ expansion, and then describing the crossover by calculating the accompanying scaling function, is bound to fail.

However, a certain extension of Amit and Goldschmidt's "generalized minimal subtraction" procedure [9] has proven to be most successful in a variety of interesting crossover scenarios [10-12]. Indeed, by refraining from any $\left(d_{c}-d\right)$ expansion, we have demonstrated that the crossover between fixed points of different upper critical dimension can be consistently incorporated into the formalism [13]. The essential point is that one has to assure that the renormalization constants take account of the UV poles for any value of the anisotropy parameter $g_{0}$, including the limit $g_{0} \rightarrow \infty$. Thus the $Z$-factors become functions of both the anharmonic coupling and the additional mass $g_{0}$. By keeping the full dimension dependence of the corresponding residua, a smooth interpolation between the different scaling regimes is achieved (for details we refer to our forthcorning paper [13]). The prize that we have to pay is that i) there is no a priori small expansion parameter, and ii) the flow equations require a numerical solution.

Thus we define renormalized fields $\phi=Z_{\phi}^{1 / 2} \phi_{0}$ and $\bar{\phi}=Z_{\phi}^{1 / 2} \widetilde{\phi}_{0}$, and dimensionless renormalized parameters $r=Z_{s}^{-1} Z_{r}\left(r_{0}-r_{0 c}\right) \mu^{-2}, \quad c^{2}=Z_{\phi} Z_{c}^{-1} c_{0}^{2}, \quad g=Z_{\phi}^{-1 / 2} Z_{c}^{-1 / 2} Z_{g} g_{0} \mu^{-1}$, and $u=Z_{\phi}^{-3 / 2} Z_{u} u_{0} B_{d}^{1 / 2} \mu^{(d-6) / 2}$, where $\mu$ is an arbitrary momentum scale, $B_{d}=\Gamma(4-d / 2) /$ $/(4 \pi)^{d / 2}$ is a geometric factor, and $r_{0 \mathrm{e}}$ denotes the fluctuation-induced shift of the percolation threshold,

$$
r_{0 \mathrm{c}}=\left[\frac{u_{0}^{2} c_{0} B_{d}}{(d-4)(6-d)} I_{13}^{d}\left(g_{0} / \sqrt{r_{0 \mathrm{c}}}\right)\right]^{2 /(6-d)} .
$$

Here we defined $I_{m n}^{d}(g)=\int_{0}^{1} x^{m / 2-1}\left(1+x g^{2}\right)^{(d-m-n) / 2} \mathrm{~d} x$, with the limits $I_{m n}^{d}(0)=2 / m$, and $\lim _{g \rightarrow \infty}\left[g^{m} I_{m n}^{d}(g)\right]=\Gamma(m / 2) \stackrel{0}{\Gamma}((n-d) / 2) / \Gamma((m+n-d) / 2)$.

The renormalization group (RG) equation explicitly takes advantage of the scale 
invariance of the system near the percolation threshold. More precisely, we observe that the bare pair correlation functions do not depend on the arbitrary renormalization scale $\mu$. By introducing Wilson's flow functions (we list the explicit one-loop results here; the symbol $\left.\right|_{0}$ indicates that the all the derivatives are to be taken at fixed bare parameters)

$$
\begin{aligned}
& \left\{\begin{array}{l}
\zeta_{\phi}=\left.\mu \frac{\partial}{\partial \mu}\right|_{0} \ln Z_{\ddagger}=\frac{v}{8}\left[1-\frac{I_{35}^{d}(g)}{I_{17}^{d}(g)}\right], \\
\zeta_{r}=\left.\mu \frac{\partial}{\partial \mu}\right|_{0} \ln \frac{r}{r_{0}-r_{0 \mathrm{c}}}=-2+\frac{3 v}{8}+\frac{v}{8} \frac{I_{35}^{d}(g)}{I_{17}^{d}(g)},
\end{array}\right. \\
& \zeta_{c}=\left.\mu \frac{\partial}{\partial \mu}\right|_{0} \ln \frac{c}{c_{0}}=-\frac{v g^{2}(d-8)}{16}\left[2 \frac{I_{37}^{d}(g)}{I_{17}^{d}(g)}-\frac{I_{55}^{d}(g)}{I_{17}^{d}(g)}\right], \\
& \zeta_{g}=\left.\mu \frac{\partial}{\partial \mu}\right|_{0} \ln \frac{g}{g_{0}}=-1-\zeta_{\phi}+\zeta_{c}+\frac{v}{4}\left[1-\frac{I_{35}^{d}(g)}{I_{17}^{d}(g)}\right] \text {, } \\
& \zeta_{u}=\left.\mu \frac{\partial}{\partial \mu}\right|_{0} \ln \frac{u}{u_{0}}=\frac{d-6}{2}+\frac{13 v}{16}+\frac{3 v}{16} \frac{I_{35}^{d}(g)}{I_{17}^{d}(g)},
\end{aligned}
$$

one arrives at the following $R G$ equation for the renormalized two-point vertex function

$$
\left[\mu \frac{\partial}{\partial \mu}+\sum_{a=\{r, c, g, u\}} \zeta_{a} a \frac{\partial}{\partial a}+\zeta_{\phi}\right] \Gamma_{11}(\mu, r, c, g, u, q, \omega)=0 .
$$

The RG equation (8) is solved by introducing the characteristics $a(l)$, which define the running parameters and couplings. They are given by the solutions of $l \mathrm{~d} a(l) / \mathrm{d} l=\zeta_{a}(l) a(l)$, with $a(1)=a$. Defining the dimensionless vertex function $\widehat{\Gamma}_{11}$ according to $\Gamma_{11}(\mu, r, c, g, u, \boldsymbol{q}, \omega)=\mu^{2} \widehat{\Gamma}_{11}\left(r, v, \boldsymbol{q} / \mu, g \omega / c \mu, \omega^{2} / c^{2} \mu^{2}\right)$, the solution of eq. (8) reads

$$
\Gamma_{11}(\mu, r, c, g, u, \boldsymbol{q}, \omega)=\mu^{2} l^{2} \exp \left[\int_{1}^{l} \zeta_{\phi}\left(l^{\prime}\right) \mathrm{d} l^{\prime} / l^{\prime}\right] \widehat{\Gamma}_{11}\left(r(l), v(l), \frac{\boldsymbol{q}}{\mu l}, \frac{g(l) \omega}{c(l) \mu l}, \frac{\omega^{2}}{c(l)^{2} \mu^{2} l^{2}}\right) .
$$

Here we have introduced an effective anharmonic coupling $v=u^{2} c I_{17}^{d}(g)$, which is finite in both limits, $g \rightarrow 0$ and $g \rightarrow \infty$. The flow of the running coupling $l \mathrm{~d} v(l) / \mathrm{d} l=\beta_{v}(l)$ is given by the corresponding $\beta$-function $\beta_{v}=\mu \partial v /\left.\partial \mu\right|_{0}$. The flow parameter $l$ may be considered as describing the effect of a scaling transformation upon the system. The theory becomes scale-invariant near a fixed point $v^{*}$, defined as a zero of the $\beta$-function, and will yield the correct asymptotic behavior, if it is infrared-stable.

We now turn to study eq. (9) in the vicinity of such a fixed point $v^{*}$; introducing $\zeta_{a}^{*}=$ $=\zeta_{a}\left(v=v^{*}\right)$ we find that $\Gamma_{11}$ is a generalized homogeneous function

$$
\Gamma_{11}(\mu, r, c, g, u, \boldsymbol{q}, \omega) \propto \mu^{2} l^{2+\xi_{3}^{*}} \widehat{\Gamma}_{11}\left(r l^{\xi_{*}^{*}}, v^{*}, \frac{\boldsymbol{q}}{\mu l}, \frac{g / c \mu \omega}{l^{1+\xi_{c}^{*}-\xi_{g}^{*}}}, \frac{\omega^{2} / c^{2} \mu^{2}}{l^{2\left(1+\xi_{c}^{*}\right)}}\right) .
$$

Using an appropriate matching condition, we can now map the asymptotic theory with manifest infrared divergences, onto a region in parameter space where the anharmonic coupling is finite. $E . g$., with the choice $l=q / \mu$, we arrive at the following self-affine scaling form:

$$
\Gamma_{11}(\mu, r, c, g, u, q, \omega) \propto q^{2-\eta_{\perp}} \widehat{\Gamma}_{11}\left(\frac{r}{(q / \mu)^{1 / \nu_{\perp}}}, v^{*}, 1, \frac{g / c \mu \omega}{(q / \mu)^{2}}, \frac{\omega^{2} c^{2} \mu^{2}}{(q / \mu)^{2 z(1-\lrcorner)}}\right),
$$


TABLE I. - Fixed-point values for the couplings $v(l)$ and $\widetilde{g}(l)$ at the isotropic (I), Gaussian isotropic (GI), directed (D), and Gaussian directed (GD) fixed points, respectively, and the corresponding values of the four independent critical exponents.

\begin{tabular}{lllclll}
\hline Fixed point & $v^{*}$ & $\tilde{g}^{*}$ & $\eta_{\perp}$ & $\nu_{\perp}^{-1}$ & $z$ & \multicolumn{1}{l}{ ( } \\
\hline GI & 0 & 0 & 0 & 2 & 2 & $1 / 2$ \\
I & $\frac{4}{7}(6-d)$ & 0 & $-\frac{6-d}{21}$ & $2-\frac{5(6-d)}{21}$ & $2-\frac{6-d}{21}$ & $\frac{1-(6-d) / 21}{2-(6-d) / 21}$ \\
GD & 0 & 1 & 0 & 2 & 2 & $1 / 2$ \\
D & $\frac{2}{3}(5-d)$ & 1 & $-\frac{5-d}{12}$ & $2-\frac{5-d}{4}$ & $2-\frac{5-d}{12}$ & $\frac{1-(5-d) / 6}{2-(5-d) / 12}$ \\
\hline
\end{tabular}

where we have defined four independent critical exponents according to $\eta_{\perp}=-\zeta_{\zeta}^{*}, \nu_{\perp}=$ $=-1 / \zeta_{r}^{*}, z=1+\zeta_{c}^{*}-\zeta_{g}^{*}$, and $z \Delta=-\zeta_{g}^{*}$. Here, $\eta_{\perp}$ and $\nu_{\perp}$ correspond to the two independent indices familiar from the theory of static critical phenomena. The «dynamic» exponent $z$ is related to the anisotropic scaling behavior. Finally, $\Delta$ is a positive crossover exponent describing the transition from isotropic to directed percolation. In the asymptotic limit of directed percolation, $g \rightarrow \infty$, the second scaling variable disappears, and the scaling behavior is described by the three exponents $\eta_{\perp}, \nu_{\perp}$, and $z$.

Similarly, with the choice $l=(g \omega / c \mu)^{1 /\left(1+\zeta_{c}^{*}-\zeta_{g}^{*}\right)}$ equation (10) reads

$\Gamma_{11}(\mu, r, c, g, u, \boldsymbol{q}, \omega) \propto \omega^{2-r_{\|}} \widehat{\Gamma}_{11}\left(\frac{r}{(q / \mu)^{1 / \nu \mid}}, v^{*}, \frac{q / \mu}{(g \omega / c \mu)^{1 / z}}, 1, \frac{\omega^{2} / c^{2} \mu^{2}}{(g \omega / c \mu)^{2(1-\lrcorner)}}\right)$,

where $2-\eta_{\|}=\left(2-\eta_{\perp}\right) / z$ and $\nu_{\|}=z \nu_{\perp}$. Moreover, $l=r^{-1 / \zeta_{*}^{*}}$ leads to

$$
\Gamma_{11}(\mu, r, c, g, u, \boldsymbol{q}, \omega) \propto r^{\gamma} \widehat{\Gamma}_{11}\left(1, v^{*}, \frac{q}{\mu} r^{-\nu_{\perp}}, \frac{g \omega}{c \mu} r^{-\nu_{\|}}, \frac{\omega^{2}}{c^{2} \mu^{2}} r^{-2 \nu_{\perp}(1-1)}\right),
$$

where the exponent $\gamma$ is related to $\nu_{\perp}$ and $\nu_{\|}$via $\gamma=\nu_{\perp}\left(2-\eta_{\perp}\right)=v_{\|}\left(2-\eta_{\|}\right)$. In the special case of isotropic percolation, the scaling relations simplify considerably, describing self-similar scaling with only two independent critical exponents $\eta=-\zeta_{\phi}^{*}$ and $\nu=$ $=-1 / \zeta_{r}^{*}$.

In the two limiting cases of isotropic and directed percolation, we can explicitly determine the critical indices to one-loop order. For $g_{0}=0$ and $d<d_{\mathrm{c}}^{\mathrm{I}}=6$, one finds a stable, non-trivial fixed point $v_{\mathrm{I}}^{*}=4(6-d) / 7$, while for $d \geqslant 6$ the Gaussian fixed point $v_{\mathrm{GI}}^{*}=0$ is approached. In the opposite case, $g_{0} \rightarrow \infty$, the nontrivial directed fixed point is stable only if $d<d_{\mathrm{c}}^{\mathrm{D}}=5$, while for $d=D+1 \geqslant 5$ the mean-field exponents of the Gaussian fixed point $v_{\mathrm{GD}}^{*}$ apply. The fixed-point values and the corresponding independent critical indices are collected in table I (there the coupling $\widetilde{g}=g /(1+g)$ was defined). The other critical exponents may be inferred from the scaling relations.

Thus we have demonstrated that both the self-similar and the self-affine scaling behavior are within the scope of the present theory, at least for dimensions $d<5$; for $5<d \leqslant 6$ the model is not renormalizable in the directed limit, and simply characterized by the exponents corresponding to the Gaussian fixed point $v_{\mathrm{GD}}^{*}$, with logarithmic corrections for $d=5$. This 


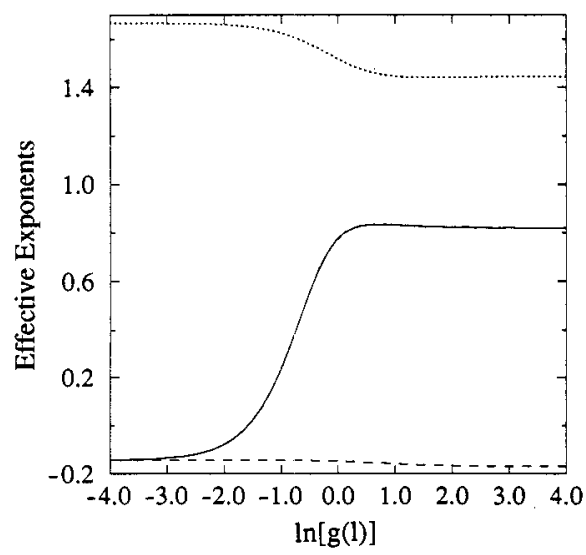

Fig. 1. - Effective exponents $\eta_{\| \text {eff }}(l)$ (solid), $\eta_{\perp \text { eff }}(l)$ (dashed), and $\gamma_{\text {eff }}(l)$ (dotted) for the connectivity as functions of the running anisotropy parameter $g(l)$ for a fixed initial value of the coupling constant $v(1)=v_{1}^{*}$.

again emphasizes the fact that no expansion with respect to a fixed upper critical dimension can be applied consistently.

The interchange from self-similar to self-affine scaling is most conveniently described by effective exponents for the pair-correlation function. Using the zero-loop result for the two-point vertex function

$$
\Gamma_{11}(r, \boldsymbol{q}, \omega)=\mu^{2} l^{2} \exp \left[\int_{1}^{l} \zeta_{\phi}\left(l^{\prime}\right) \mathrm{d} l^{\prime} / l^{\prime}\right]\left[r(l)+\left(\frac{q}{\mu l}\right)^{2}+\frac{\omega^{2}}{\mu^{2} l^{2} c(l)^{2}}+2 i \frac{\omega g(l)}{\mu l c(l)}\right),
$$

we specialize to $r=0$ and $\boldsymbol{q}=\mathbf{0}$ and define (compare eq. (12))

$$
2-\eta_{\| \text {eff }}(\omega)=\frac{\mathrm{d} \ln \sqrt{\left|\Gamma_{11}(0,0, \omega)\right|^{2}}}{\mathrm{~d} \ln \omega} .
$$

Using $\left|\omega^{2} / \mu^{2} l^{2} c(l)^{2}+2 i \omega g(l) / \mu l c(l)\right|^{2}=1$ yields $2-\eta_{\| \text {eff }}(l)=\left[2+\zeta_{\phi}(l)\right] \mathrm{d} \ln l / \mathrm{d} \ln \omega . \operatorname{Sim}-$ ilarly, in the case $r=\omega=0$ (see eq. (11)), let

$$
2-\eta_{\perp \text { eff }}(q)=\frac{\mathrm{d} \ln \Gamma_{11}(0, q, 0)}{\mathrm{d} \ln q},
$$

which reduces to $2-\eta_{\perp \text { eff }}=2+\zeta_{\phi}(l)$, if $(q / \mu l)^{2}=1$ is inserted. Finally, considering $\boldsymbol{q}=\mathbf{0}$ and $\omega=0$, we introduce (compare eq. (13))

$$
\gamma_{\mathrm{eff}}(r)=\frac{\mathrm{d} \ln \Gamma_{11}(r, 0,0)}{\mathrm{d} \ln r}
$$

and choosing the matching condition $r(l)=1$, we find $\gamma_{\text {eff }}(l)=-\left[2+\zeta_{\phi}(l)\right] / \zeta_{r}(l)$.

The flow of the effective exponents $\eta_{\text {ileff }}(l), \eta_{\perp \text { eff }}(l)$, and $\gamma_{\text {eff }}(l)$ in $d=D+1=3$ dimensions (with $\mu=1$ ) is depicted in fig. 1 , with the initial value for the coupling $v(1)=v_{\mathrm{I}}^{*}$ of the isotropic scaling fixed point. The dependence on the anisotropy scale $g$ was eliminated by plotting $v s$. the scaling variable $\ln g(l)$; the graphs corresponding to different initial values $g(1)$ then all collapse onto one master curve. The most important conclusion to be drawn from fig. 1 is that the anisotropy scale, at which the crossover occurs, considerably differs for the effective exponent defined above! $\eta_{\| \text {eff }}$ starts to cross over from the isotropic to the directed fixed-point value already at $\ln g\left(l_{\text {cross }}\right) \approx-0.8$, whereas $\gamma_{\text {eff }}$ shows this crossover at 
$\ln g\left(l_{\text {cross }}\right) \approx-0.2$, and $\eta_{\perp \text { eff }}$ only at $\ln g\left(l_{\text {cross }}\right) \approx+0.8$. Note that the sizeable change of $\eta_{\| \text {eff }}$ is already apparent at mean-field level, where it acquires the values 0 and 1 in the isotropic and directed limit, respectively. However, a crossover of the exponents $\eta_{\perp \text { eff }}$ and $\gamma_{\text {eff }}$ requires the $\zeta$-functions at least on the one-loop level.

We remark that a calculation of the above crossover features has not been possible up to this present work. Of course, the exponents for the limits of both isotropic and directed percolation have been determined to a much higher accuracy than is provided in our one-loop approximation [2-5]. However, our aim was rather to describe the crossover features, and we believe that the crossover loci should not be affected too severely by, say, higher orders of perturbation theory. The expectation that our renormalized mean-field theory, accompanied with the one-loop results for the flowing parameters, in a reasonably good approximation, is based on the experience that amplitude functions are usually smooth and enter the results less sensitively than the exponent functions. In fact, our method was designed to incorporate the entire crossover behavior into the Wilson flow functions. The proposed approach to crossover phenomena, which is an extension of Amit and Goldschmidt's "generalized minimal subtraction" scheme [9], should thus be applicable to a large variety of crossover phenomena. It would, therefore, be of considerable interest to compare our predictions concerning the crossover scales of the different effective exponents with the outcome of precise computer simulations and/or experiments in order to estimate the quality of our approximations also in different situations, where numerical simulations are either very cumbersome or not feasible at all.

This work has been supported by the Deutsche Forschungsgemeinschaft (DFG) under Contracts No. Fr. 850/2-1.2, Ta. 177/1-1, and Schw. 348/4-2.

\section{REFERENCES}

[1] Mandelbrot B. B., The Fractal Geometry of Nature (Freeman, San Francisco) 1982; Feder J., Fractals (Plenum, New York, N.Y.) 1988.

[2] See, e.g., Essam J. W., Rep. Prog. Phys., 43 (1980) 834; Stauffer D. and Aharony A., Introduction to Percolation Theory, 2nd edition (Taylor and Francis, London) 1992.

[3] See, e.g., Kinzel W., in Percolation Structures and Processes, Ann. Isr. Phys. Soc., Vol. 5, edited by G. Deutscher, R. ZALlen and J. AdLer (Bar-Ilan University) 1983, p. 425.

[4] Cardy J. L. and Sugar R. L., J. Phys. A, 13 (1980) L-423.

[5] Benzoni J. and Cardy J. L., J. Phys. A, 17 (1984) 179.

[6] AмIт D. J., Field Theory, the Renormalization Group, and Critical Phenomena, 2nd edition (World Scientific, Singapore) 1984.

[7] Janssen H. K., Z. Phys. B, 23 (1976) 377; Bausch R., Janssen H. K. and Wagner H., Z. Phys. $B, 24$ (1976) 113.

[8] t'Hoof G. and Veltuan M., Nucl. Phys. B, 44 (1972) 189.

[9] Amit D. J. and Goldschmidt Y. Y., Ann. Phys. (N.Y.), 114 (1978) 356.

[10] Lawrie I. D., J. Phys. A, 14 (1981) 2489; 18 (1985) 1141.

[11] Frey E. and Schwabl F., Phys. Rev. B, 42 (1990) 8261; 43(1991) 833.

[12] Täuber U. C. and Schwabl F., Phys. Rev. B, 46 (1992) 3337; 48 (1993) 186.

[13] Frey E., Täuber U. C. and Schwabl F., to be published in Phys. Rev. B (1994). 